\title{
Some Numerical Evidence Concerning the Uniqueness of the Markov Numbers
}

\author{
By D. Rosen and G. S. Patterson, Jr.*
}

\begin{abstract}
A Markov triple is a set of three positive integers satisfying the diophantine equation $\left(x^{2}+y^{2}+z^{2}=3 x y z\right)$. The maximum of the triple is called a Markov number. Although all Markov triples can be generated from the triple $(1,1,1)$, it is not known whether it is possible to obtain $\left(p, a_{1}, b_{1}\right)$ and $\left(p, a_{2}, b_{2}\right)$, where $p$ is the same Markov number for both triples. All Markov numbers not exceeding 30 digits were computed without turning up a duplication, lending some credence to the conjecture that the Markov numbers are unique.
\end{abstract}

The triple of positive integers $(p, a, b)$ which satisfy the diophantine equation

$$
x^{2}+y^{2}+z^{2}=3 x y z
$$

is called a Markov triple and the maximum of the triple is called a Markov number. We shall always write the triple so that the first entry is the Markov number. It turns out, however, [1, p. 28], that every member of the triple is a Markov number for some triple.

The solutions to (1) are well known (see [1], [2], [3], or [5]) in the sense that, given any Markov triple, the others can be generated from it. In particular, if $(p, a, b)$ is a Markov triple, then two new Markov triples, $\left(p_{1}, a, p\right)$ and $\left(p_{2}, p, b\right)$, are obtained where

$$
p_{1}=3 p a-b, \quad p_{2}=3 p b-a
$$

and $p_{1}, p_{2}$ are both greater than $p$. Thus, $p_{1}, p_{2}$ are Markov numbers. A third possible recursion value, $p^{\prime}=3 a b-p$, turns out to be a smaller Markov number.

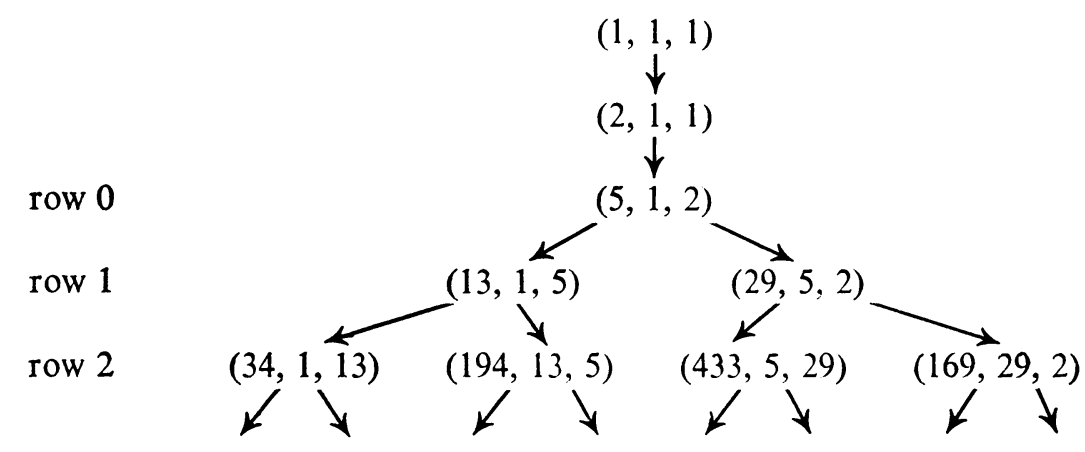

Received July 8, 1970, revised February 19, 1971.

AMS 1969 subject classifications. Primary 1003, 1010.

Key words and phrases. Markov number, Markov triple, diophantine equation, binary tree, node, branch, bit string, preorder traversal algorithm.

* The first author acknowledges with much thanks that some of the work on this problem was done while on a sabbatical leave from Swarthmore College under a special grant from the Sloan Foundation to Swarthmore College for the improvement of teaching in the sciences.

Copyright $\odot$ 1971, American Mathematical Society 
By starting with the initial Markov triple, $(1,1,1)$, all triples are found on the binary tree generated by (2). The first few branches are as in the diagram above. Observe that the tree bifurcates beginning with $(5,1,2)$.

The question we hoped a machine computation would answer was posed by Frobenius [3] and repeated by Cassels [1]. Essentially, they ask whether it is possible for the same Markov number to occur in two different nodes of the tree; i.e., is it possible to obtain two different triples $\left(p, a_{1}, b_{1}\right)$ and $\left(p, a_{2}, b_{2}\right)$ where $p$ is the same Markov number for both triples? Observe that, if $a_{1}=a_{2}$ or $a_{1}=b_{2}$, then the triples are necessarily identical except for the order in which the elements are written. This follows immediately, for if $a_{1}=b_{2}$ say, then

$$
p^{2}+a_{1}^{2}+b_{1}^{2}=3 p a_{1} b_{1} \text { and } p^{2}+a_{2}^{2}+b_{2}^{2}=3 p a_{2} b_{2}
$$

imply

$$
b_{1}^{2}-a_{2}^{2}=3 p a_{1}\left(b_{1}-a_{2}\right) .
$$

Either $b_{1}=a_{2}$, in which case the triples are identical, or $b_{1}+a_{2}=3 p a_{1}$; hence $a_{2}=3 p a_{1}-b_{1}$. Now, according to the recursion formulae (2), $3 p a_{1}-b_{1}=p_{1}>p$; hence $a_{2}>p$, which contradicts the assumption that $p$ is the Markov number for $\left(p, a_{2}, b_{2}\right)$. A similar argument holds if $a_{1}=a_{2}$.

A preliminary search for possible duplications was made by Professor E. R. Mullins, Jr., while on leave at the University of Essex, England, on the ICT-1900. The tree was generated by computing all members of each row. However, by the eighth row ( $2^{8}$ entries), Markov numbers of 56 digits were appearing. The software used limited the precision of the computation to less than 20 digits. Nevertheless, the evidence indicated that no duplications would occur.

More recently, we computed all the Markov numbers not exceeding 30 digits, finding 893 of them. In order to accomplish this, it was necessary to calculate the triples through row 70 before exhausting all Markov numbers with at most 30 digits.

Empirically, we noticed that there are roughly $N^{2}$ Markov numbers not exceeding $N$ digits.

\begin{tabular}{cc}
$N$ Digits & Markov Numbers $\leqq N$ Digits \\
\hline 5 & 31 \\
10 & 107 \\
15 & 231 \\
20 & 404 \\
25 & 624 \\
30 & 893
\end{tabular}

It is evident that the Markov numbers are spread rather thinly among the integers. We point out as an amusement that the even subscripted Fibonacci numbers are Markov numbers obtained on the extreme left branch of the tree. If the Fibonacci numbers are denoted by $u_{0}, u_{1}, u_{2}, \cdots, u_{n}, \cdots$, then the Markov numbers are $u_{0}=1$, $u_{2}=2, u_{4}=5, u_{6}=13, u_{8}=34$, etc.

The present computation was made on an IBM 1130 at Swarthmore College using the IBM commercial subroutine package for handling the decimal arithmetic 
plus a set of ASSEMBLER subroutines written by us to perform bit string manipulation. The coding to generate the tree was done in FORTRAN. The branches of the tree were calculated by using the recursion formulae (2) and the branch was terminated when the computed Markov number exceeded $N$ digits. The tree was established by a preorder traversal algorithm [4, p. 315]. As an example, we display the tree structure for all four-digit Markov numbers.

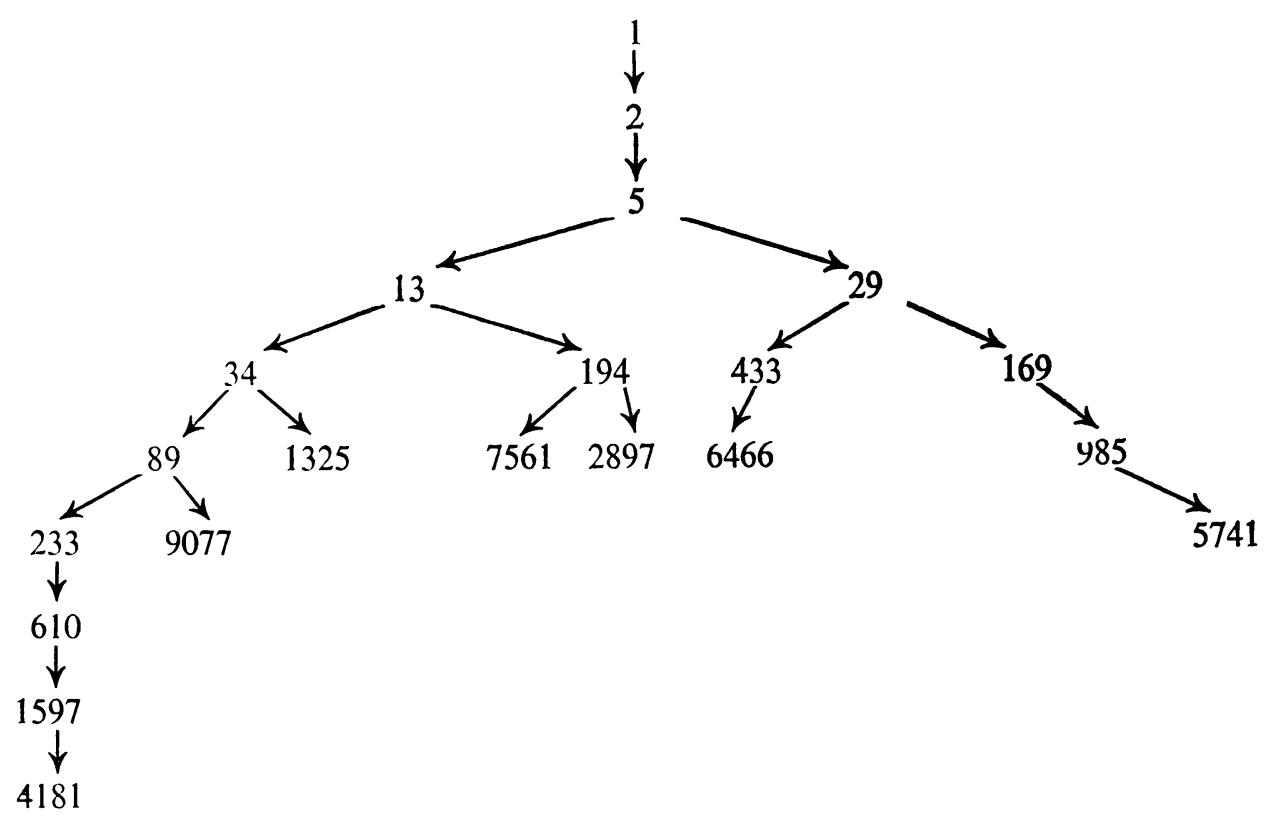

The entire tree structure was also listed by identifying each node by a bit string as well as by a table of links between nodes. These tables are available upon request as well as a list of all the 30-digit Markov numbers in ascending order. We are pleased to report that no duplications were uncovered.

We point out that knowing the uniqueness of the Markov numbers has an importance in the theory of quadratic forms [2, pp. 100-106].

Department of Mathematics

Swarthmore College

Swarthmore, Pennsylvania 19081

Department of Engineering

Swarthmore College

Swarthmore, Pennsylvania 19081

1. J. W. S. Cassels, Introduction to Diophantine Approximation, Cambridge Tracts in Math. and Math. Phys., no. 45, Cambridge Univ. Press, New York, 1957. MR 19, 396. Ill., 1930.

2. L. E. Dickson, Studies in the Theory of Numbers, Univ. of Chicago Press, Chicago,

3. G. Frobenius, "Úber die Markoffschen Zahlen," S.-B. Preuss. Akad. Wiss., v. 1913, pp. $458-487$.

4. D. E. KNUTH, The Art of Computer Programming. Vol. I. Fundamental Algorithms, Addison-Wesley, Reading, Mass., 1968.

5. A. MARKOFF, "Sur les formes quadratiques binaires indefinées," Math. Ann., v. 15, 1879, pp. 381-409. 